Corrigendum

\title{
Corrigendum to "Security Measurement in Industrial IoT with Cloud Computing Perspective: Taxonomy, Issues, and Future Directions"
}

\author{
Sahar Shah, ${ }^{1}$ Mahnoor Khan, ${ }^{2}$ Ahmad Almogren $\left(\mathbb{D},{ }^{3}\right.$ Ihsan Ali, ${ }^{4}$ Lianwen Deng $\mathbb{D},{ }^{5}$ \\ Heng Luo, ${ }^{5}$ and Muazzam A. Khan ${ }^{6}$ \\ ${ }^{1}$ Department of Electronics, Quaid-i-Azam University, Islamabad, Pakistan \\ ${ }^{2}$ Department of Physics, Government Post Graduate College, Nowshera, Pakistan \\ ${ }^{3}$ Chair of Cyber Security, Computer Science Department, College of Computer and Information Sciences, King Saud University, \\ Riyadh, Saudi Arabia \\ ${ }^{4}$ Faculty of Computer Science and IT, University of Malaya, Kuala Lampur, Malaysia \\ ${ }^{5}$ School of Physics and Electronics, Central South University, Changsha, China \\ ${ }^{6}$ Department of Computer Science, Quaid-i-Azam University, Islamabad, Pakistan
}

Correspondence should be addressed to Ahmad Almogren; ahalmogren@ksu.edu.sa and Ihsan Ali; ihsanalichd@siswa.um.edu.my Received 16 December 2020; Accepted 16 December 2020; Published 30 December 2020

Copyright (c) 2020 Sahar Shah et al. This is an open access article distributed under the Creative Commons Attribution License, which permits unrestricted use, distribution, and reproduction in any medium, provided the original work is properly cited.

In the article titled "Security Measurement in Industrial IoT with Cloud Computing Perspective: Taxonomy, Issues, and Future Directions" [1], there was an error in the third and sixth affiliations. The corrected affiliation list is shown above. In addition, the Acknowledgements section should be updated as follows:

"The authors are grateful to the Deanship of Scientific Research, King Saud University for funding through Vice Deanship of Scientific Research Chairs and partially supported by the Faculty of Computer Science and Information Technology, University of Malaya, under Postgraduate Research Grant PG035-2016A."

\section{References}

[1] S. Shah, M. Khan, A. Ahmad et al., "Security measurement in industrial iot with cloud computing perspective: taxonomy, issues, and future directions," Scientific Programming, vol. 2020, Article ID 8871315, 31 pages, 2020. 